\title{
Vendor managed forecasting: A case study of small enterprise
}

\author{
Atul Borade ${ }^{1}$, Satish Bansod ${ }^{2}$ \\ ${ }^{1}$ St Vincent Pallotti College of Engineering and Technology (INDLA); \\ ${ }^{2}$ Ram Meghe Institute of Research and Technology (INDLA) \\ atulborade@,rediffmail.com; satishbansod@,rediffmail.com
}

Received November 2008

Accepted March 2009

\begin{abstract}
Enterprises use supply chain management practices for improving business or supply chain performance. It is observed that supply chain technologies like VMI are now becoming an integral part of enterprise's strategy. Even small and medium enterprises can adopt this practice and improve the performance of supply chain. This paper discusses vendor managed forecasting with the help of case study. It shows how a small enterprise improves supply chain performance by using demand related information obtained from retailer. The results obtained in the study shows that vendor managed forecasting in supply chain reduces the demand variation and improves inventory management significantly.
\end{abstract}

Keywords: supply chain management, demand forecasting, performance measures, vendormanaged inventory, small and medium enterprise

\section{Introduction}

In recent years, many enterprises have been compelled to share demand and inventory information with their suppliers and customers to get the competitive advantage (Disney \& Towill, 2003). Vendor Managed Inventory (VMI) is one of such information sharing mechanisms adopted by organizations. In VMI, the vendor or supplier is given the responsibility of managing the customer's stock, based on the shared information between them (Jung et al., 2004). Vendor coordinates and integrates all supply chain activities into a seamless process. As a 
result of VMI adoption drastic improvements are observed in supply chain and business performance.

Several performance measures have been reported in the literature to measure different aspects of the supply chain performance. Usually the measures are inventory levels, service levels, order fulfillment time, total supply chain cost, forecasting accuracy, supply chain flexibility, fill rates etc. A careful study of these measures shows that the common denominator is accurate demand forecasting. If the forecast is not accurate, the quantity ordered by retailers may not reflect the demand for the period and the errors in retailers forecast may pass to the supplier in form of distorted orders (Zhao et al., 2003). Therefore forecasting results are very influential in making managerial decisions and evaluating performance of the company (Haq \& Kannan, 2006).

It has been observed that, to improve the accuracy of forecasts there is an incessant need to interact with supply chain partners and share right kind of information at right time. Enterprises adopt latest information technology tools to share real-time information. However, many Enterprises experiences difficulty in adoption of IT tools. Moreover, it is found that adoption is more difficult for small and medium enterprises. In a dedicated supply chain, where small and medium enterprises are under vendor managed inventory pact with retailers; the retailers and managers at SMEs do not have a capacity and capability of forecasting the demand accurately. Rudberg et al. (2002) have suggested that collaboration and demand information sharing with supply chain partners to establish a joint forecast; could reduce demand uncertainty within the supply chain. Taking the motivation we decided to adopt Vendor managed Inventory, specifically Vendor Managed Forecasting practice in a small scale Industry. It is known that for adopting VMI, organizations use supply chain software. Supply chain software's have an inbuilt algorithms based on statistical forecasting models which assist organizations in forecasting. The case company was not able to afford the software. Therefore, it was decided to go for traditional VMI and develop manual forecasts based on statistical models. The accuracy of the forecast highly depended on the information provided by retailers about the demand. So, information sharing mechanism was also developed.

In this paper we have studied vendor managed forecasting for a small-scale enterprise located at the central part of India. The objective of study is to show 
how that information sharing mechanism helps a small-scale enterprise to make a better forecast and improve the supply chain performance. In the process we have compared the forecasts obtained from different statistical models .The remaining organization of the paper is as follows. In the section 2, we have presented the literature that focuses on quantitative forecasting models. Section 3, describes the case study and action research methodology. Section 4 ponders performance measurement. Section 5 discusses the results and finally in Section 6 conclusions are drawn.

\section{Review of literature}

Vendor managed inventory demands vendor to perform host of activities. Activities start from arranging raw material from suppliers and ends when finished goods are delivered to customers. Production schedule, capital budgeting, manpower forecasting, marketing activities heavily depend upon the expected future sales. Hence, without any doubt, first and foremost activity is demand forecasting. Multinational companies use ERP or any other supply chain software for demand forecasting. In the case company software was not available so it was decided to make a forecast using traditional methods of forecasting. Traditional or statistical methods of forecasting are easy to understand and implement. However, there are varieties of models, which could be used. Therefore we tried to understand the various methods and their applications. We begin our literature review on statistical forecasting models by referring the famous book of (Box et al., 2004). Authors have provided deeper insights on the theory and practical applications of statistical models. Demand was assumed either linear or cyclic in nature. Initially, a general linear stochastic model was studied. Then, the properties and types of autoregressive moving average models (ARMA) were studied in detail. We know that certain observation exhibits non-stationary behavior; hence for these classes of stochastic time series, autoregressive integrated moving average (ARIMA) models were studied along with their properties.

We also investigated the relative capabilities of different forecasting methods and the effects of various horizon lengths and data periods. Holt et al. (1963); Harrison (1965); Raveh (1980) studied the issue of short-term sales forecasting with respect to the lead-time in inventory control problems. The impact of forecast on retail sales and retail stock was evaluated, and it was concluded that accurate sales forecast are important in effective inventory management. Authors also recognized 
the difference and effect of aggregate and disaggregate sales forecasting on inventory management. Whang and Huang (2007) identified effect of data type, horizon length, and data period factors on the performance of a forecasting model. It was found that even though data type is inherently dictated by chosen data, decision maker could specify controllable factors such as horizon length and data period.

Kurawarwala and Matsuo (1998) found that the traditional short term and medium term forecasting methods does not take in to account the shorter product life cycles. In shorter life cycle environments, the scarcity of data for any given product is offset by availability of data on prior similar products. Authors proposed a forecasting method that extracts relevant information from prior products and that supplements key information for forecasting. They empirically validated the model and show that the accuracy of the forecast for multiple periods is better than that of ARIMA models. For time series with a long history, Sharda and Patil (1992) showed that sophisticated time series methods such as decomposition models and Box-Jenkins models could provide compatible results.

Modeling seasonal time series is a challenging task. One popular approach to deal with seasonal data is to remove the seasonal component first, and then estimate other components. Many practitioners in various forecasting applications have satisfactorily adopted this practice of seasonal adjustment (Chu \& Zhang, 2003). Research by Withycombe (1989) as cited in Dekkar et al. (2004) showed a different approach for seasonal adjustment. Instead of updating seasonal indices, like in the Holt-Winters' method, author used the improved seasonal estimates to deseasonalize each series in the group, before extrapolation by Double Exponential Smoothing, and reseasonalize afterwards. Bunn and Vassilopoulos (1999) as cited in Dekkar et al. (2004) addressed the issue of estimating seasonal indices for multi-item, short-term forecasting, based upon both individual time series estimates and groups of similar time series. This development of the joint use of individual and group seasonal estimation was extended in two directions. One method was derived from the procedures developed for combining forecasts. The second method employed Stein Rules to obtain shrinkage estimates of seasonal components. Authors showed that combined indices offer highest improvement in forecasting performance. 
Yelland (2006) discussed number of stable seasonal pattern models from the literature, which have been evaluated for making forecast revisions at Sun Microsystems, Inc. Commonalities between the models are elucidated using a general theoretical framework, and a straightforward sample based mechanism is described that affords great flexibility in the design and use of stable seasonal pattern models. With simulations based on actual sales series of selected Sun products, the forecast performance of each model and its fit to the data are determined and compared with those of the others. Dekkar et al. (2004) found that standard forecasting methods that are designed to cope with seasonal demand often are no longer applicable in practice. Due to growing assortments and shorter product life cycles, demand data may show too high variation or may be insufficient to construct reliable forecast models at the individual item level. Authors presented alternative forecasting methods that are based on using demand information from a higher aggregation level and on combining forecasts. Synder et al. (2004) argued that the random error component of a demand series depends on trend and seasonal effects. They derived formulae for the mean and variance of LTD for many common forms of exponential smoothing and examined the impact of stochastic lead-times for the special case corresponding to simple exponential smoothing. These formulae ensured safety stocks adjustments according to the changes in trend or changes in season. Researchers observed that when the data is insufficient to characterize the system, grey forecasting models could give very promising results. One such application is found in (Tseng et al., 2001). Authors provided an application of grey theory to time series problems, which include seasonality. Authors proved that the $\operatorname{GM}(1,1)$ grey model is insufficient for forecasting seasonal time series. They proposed to use the ratio-to-moving average method to remove the seasonality out of seasonal time series.

Few researchers have suggested and developed tailor-made statistical models. For example, Winklhofera and Diamantopoulos (2003) presented and tested a path model of export sales forecasting behavior and performance that incorporated organizational and export-specific characteristics. The results indicated that the key determinants of export sales forecasting performance are organizational commitment, in addition to the resources devoted to the forecasting function. Frees and Millar (2004) introduced the longitudinal data mixed model, a class of models that extends the traditional two-way error components longitudinal data models with the framework of a mixed linear model. Authors developed a forecast 
model using a class of linear mixed longitudinal, or panel, data models. Forecasts were derived as special cases of best linear unbiased predictors, also known as BLUPs, and were optimal predictors of future realizations of the response. It was shown that the BLUP forecast arises from three components: (1) a predictor based on the conditional mean of the response, (2) a component due to time-varying coefficients, and (3) a serial correlation correction term. In 2007, Whang and Huang employed Taguchi technique to rank and evaluate effects of controllable forecasting factors. Initially, quantitative factors and level settings were determined later an orthogonal array was adopted to determine appropriate treatment combinations. The resulting response table of the design experiment was then analyzed in order to rank and screen controllable factors. The results showed that the proposed model permits the construction of a highly efficient forecasting model through the suggested data collection method. A different attempt can be seen in Thomassey and Fiordaliso (2006). In this paper, a model based on existing clustering technique ( $\mathrm{k}$-means algorithm) and decision tree classifier (C4.5 algorithm) was proposed. It was found suitable for estimation of new items, where no historical sales data is available. In the clustering procedure similar historical items in term of sales profiles were grouped and then, the decision tree associated future items with prototypes from their descriptive criteria. The resulting prototypes constituted the sales forecast.

Literature shows that statistical models of forecasting along with information sharing can reduce the stock outs and overstocks. It is felt that better forecasts would also help in improving supply chain performance. Getting the motivation from the studies we decided to adopt vendor managed forecasting in a small enterprise. By processing previous year data forecast from adaptive statistical models were obtained. Different models, formulas and the smoothing factors used are discussed below.

\subsection{Overview of statistical forecasting models}

A forecast is an estimate of the level of demand to be expected for a product for some period of time in future. If company know its customers past behavior, it may shed light on their future behavior. Thus, forecast is basically a guess, but by the use of certain techniques it could be more than just a guess. The objective of every forecast is to support decisions that are based on the forecast, so the 
company must clearly identify forecasting model (Biegel, 1987). In the following sections we discuss the statistical models for demand forecasting.

\section{Winter's Model.}

Winter's exponential smoothing model is used for forecasting the demand when the effects of seasonality and trend are to be taken into consideration. According to this method, three components to the model are assumed: a permanent component, a trend, and a seasonal component. Each component is continuously updated using a smoothing constant. It is applied to the most recent observation and the last estimate. In the Winter's model, it is assumed that each observation is the sum of a deseasonalized value and a seasonal index

$$
\begin{aligned}
& S_{t}=a\left(X_{t}-I_{t-L}\right)+(1-a)\left(S_{t-1}+b_{t-1}\right) \\
& b_{t}=\gamma\left(S_{t}-S_{t-1}\right)+(1-a) b_{t-1} \\
& I_{t}=B\left(X_{t}-S_{t}\right)+(1-B) I_{t-L}
\end{aligned}
$$

and forecasts are computed by the formula

$$
\mathrm{F}_{\mathrm{t}+\mathrm{m}}=\mathrm{S}_{\mathrm{t}}+\mathrm{I}_{\mathrm{t}-\mathrm{L}+\mathrm{m}}
$$

where $a, \beta, \gamma$ are general smoothing, seasonal smoothing and trend smoothing constant respectively. $\mathrm{L}$ is the length of seasonality; $\mathrm{b}_{\mathrm{t}}$ is the trend component; $\mathrm{I}$ is the seasonal adjustment factor; $S_{t}$ is the smoothed series that does not include seasonality; and $\mathrm{F}_{\mathrm{t}+\mathrm{m}}$ is the forecast $\mathrm{m}$ periods ahead. In our study best results were obtained by using $a=0.3, \beta=0.4$ and $\gamma=0.5$ (Chang, 2005)

\section{Holt's Smoothing Model}

In this model trend is incorporated in an exponentially smoothed forecast. It is also called as trend corrected exponential smoothing method. With this approach the estimates for both the average and the trend are smoothed. It requires two smoothing constants namely $a$ and B. For our study, best values were 0.2 and 0.2 respectively.

The forecast is given as,

$$
F_{t+1=} A_{t}+T_{t}
$$


where,

$$
A_{t}=a D_{t}+(1-a)\left(A_{t-1}+T_{t-1}\right)
$$

and,

$$
T_{t}=B\left(A_{t}-A_{t-1}\right)+(1-B) T_{t-1}
$$

where $A_{t}$ is exponentially smoothed average of the series in period $t, T_{t}$ is exponentially smoothed average of the trend in period $t$, $a$ is smoothing parameter for the average, $B$ is smoothing parameter for the trend and $F_{t+1}$ is forecast for period t+1 (Krajewski \& Ritzman,2000).

\section{Fourier Smoothing Model}

This model uses Fourier transforms to decompose data into its sine and cosine components. The output of the transformation represents the signal in the Fourier or frequency domain, while the input signal may be in the temporal domain or spatial domain. The Fourier transform can separate low- and high- frequency information of a data. Usually, low frequencies give information about background and overall shape and high frequencies give information about details and noise. Transformations are given as

$$
\begin{aligned}
& F(w)=\int_{-\infty}^{\infty} f(x) \cdot e^{-2 \pi i w x} d x \\
& \text { where } f(x)=\int_{-\infty}^{\infty} F(w) \cdot e^{2 \pi i w x} d w
\end{aligned}
$$

$F(w), f(x)$ correspond to the forward and inverse Fourier Transformation (Chang, 2005).

\section{Simple Exponential Smoothing Model}

This model calculates the average of a time series by giving recent demands more weights than earlier demands. Exponential smoothing method requires three items of data: the last period's forecast; demand for this period and smoothing parameter a. It is taken as 0.2 in this study. To obtain the forecast we calculate a 
weighted average of most recent demand and the forecast of last period. The equation of the forecast is,

$$
\mathrm{F}_{\mathrm{t}+1}=a \mathrm{D}_{\mathrm{t}}+(1-a) \mathrm{F}_{\mathrm{t}}
$$

Where $F$ is forecast for period, $D$ is demand for the period and $t$ is time period. (Chopra \& Meindel, 2001)

\section{Cyclical Forecasting Models}

Cyclic forecaster is specially used when the historical data shows seasonality. This model can exhibits combination of constant and seasonal component of the time series. Cyclic forecasting function is given as,

$$
\mathrm{F}=\mathrm{a}+\mathrm{u} \cos (2 \pi / N) \mathrm{t}+\mathrm{v} \sin (2 \pi / N) \mathrm{t}
$$

Where $\mathrm{F}$ is forecast, $\mathrm{a}$ is constant component of data, $\mathrm{u}$ is constant of sine function and $\mathrm{v}$ is constant of cosine function $\mathrm{N}$ is number of observations and $\mathrm{t}$ is time period (Biegel, 1987).

\section{Case Company and Action Research Methodology}

The Case Company was a small-scale enterprise located in central part of India engaged in manufacturing and distribution of bakery products. The company was a new entrant. The marketing, production and inventory management activities were not streamlined. Management was keen to improve the business performance. Management personally knew authors and was aware about author's research interest in collaborative business practices. Therefore, management contacted us to recommend some suggestions. We studied demand inventory policies of company. We found that retailers were not able to forecast the demand accurately. Many times there were stock out and excess inventory calls from retailers. In order to reduce stock outs, retailers placed exaggerated orders to the company. Each time retailers recognized excess inventory proportions; they used to put an order of fewer quantities. In order to boost the sales, company had assured retailers to take back excess finished goods. It had also promised to pay the penalty cost for every stock out. Accordingly, there were larger excess inventories at retailers.

Table 1 yields the excess and short inventory status for a single retailer for past two years. In the year, 2004-05 excess inventories were $22.27 \%$ and short 
inventory was only $2.59 \%$. Thus overall demand variance was around $25 \%$. Company was in its establishment phase; hence initially lead-time was around 6 days. Retailers were placing exaggerated ordering quantities therefore were able to satisfy the demand of end customers to greatest extent. Higher Customer Satisfaction Index, 0.771 was observed. To reduce the wastage of finished products, company changed inventory management policy. For year 2005-06, Company decided to reduce excess inventory and supply chain cost by supplying less than what is demanded by retailer. Company also managed to reduce the lead-time to 5 days by taking follow up of retailer's orders. Manager at the company personally looked at every order of the retailer and by comparing his past performance he decided the ordering quantity. This strategy reduced the percentage of excess inventory but reduced the customer satisfaction index. From Table 1 it can be seen that excess inventory proportions were reduced from 22.27 $\%$ to $13.68 \%$. The stock outs went from $2.59 \%$ to $18.94 \%$. The increased percentage of stock outs was not a problem for retailers because company was bearing the penalty cost. However, reduced Customer Satisfaction Index, 0.283 was a concern for retailers as well as company.

It was felt that a there is a need of decision support system that will assist in inventory management decisions. Identifying the need of accurate forecasts, we recommended company to develop the forecasts on behalf of retailers. We suggested going for partial Vendor managed inventory practice, where company would take up few inventory management decisions. Company was recommended to take previous two years daily sales data from the retailer. Then by analyzing data, we developed forecasts using various statistical forecasting. It was observed that product being seasonal in nature; demand follows a cyclic pattern. Therefore, a cyclic forecaster was chosen for developing forecasts.

Instead of retailer placing the order, manufacture decided the supplying quantity of retailer. The decision was taken on the basis of forecast developed from statistical methods. Each evening the retailers was contacted on telephone and was informed about the delivery. If retailer had an anticipation of additional demand then the delivery quantity was modified. Similarly, order was modified when retailer expected fewer sales. The order was generated and supplied by manufacturer while retailer gave consent to the order. To, ensure against stock out or excess stock situations, company continued the policy of take back and penalty cost. 
In this study, we have considered a two level supply chain for a single product. The product was of daily need and was manufactured daily. In general, there are various products, which are required on daily or weekly basis. Similarly, we can find supply chain networks where there is a commitment of supply of single product to single or multiple retailers. So no doubt, the results of the vendor managed forecasting could be easily extrapolated to above-mentioned supply chains.

Business or supply chain performance depends on many factors. However, in order to measure the effect of proper forecasting we would not concentrate on other factors. It was decided that if the results were good then this practice would be implemented for all retailers. We followed the results of Cyclical Forecasting Model; however we compare the results of various methods adopted in this study.

\section{Performance measurement of vendor managed forecasting}

\subsection{Forecasting accuracy}

Forecasting activities are widely performed in various areas of supply chains for predicting important supply chain management measurements such as demand volume in order management, product quality in manufacturing process, capacity usage in production management, and so on. The accuracy of forecasting has a great influence on the efficiency of SCM (Zhifang Liu et al., 2006). In order to evaluate the accuracy and performance of different forecasting models, researchers adopt verity of performance measures. The most commonly adopted evaluating measures are Absolute Error, Mean Absolute Deviation, Mean Absolute Percentage Error and Tracking Signal (Chang \& Wang, 2006; Chang et al., 2005; Alon et al., 2001; Aburto \& Weber, 2007). Hence we decided to adopt these measures. In order to find the difference in forecasted demand and actual demand we performed thorough error analysis by using following formulas

1. Absolute Error (A.E)

$$
\text { Absolute error }=\left|F_{t}-D_{t}\right|
$$

2. Mean Absolute Deviation (M.A.D.) 


$$
\mathrm{MAD}=1 / n \sum_{t=1}^{n}\left|F_{t}-D_{t}\right|
$$

3. Percent Error (P.E)

$$
\mathrm{PE}=100 *\left|\mathrm{~F}_{\mathrm{t}}-\mathrm{D}_{\mathrm{t}}\right| / \mathrm{D}_{\mathrm{t}}
$$

4. Mean Absolute Percentage Error (M.A.P.E.)

$$
\operatorname{MAPE}=1 / n \sum_{t=1}^{n}\left|F_{t}-D_{t}\right| / D_{t} \cdot 100
$$

5. Tracking Signal (T.S.)

$$
\mathrm{T} \mathrm{S}=\sum_{t=1}^{n}\left|F_{t}-D_{t}\right| / M A D_{t}
$$

where, $n$ is number of observations, $F_{t}$ is forecast for period $t, D_{t}$ is demand for period $t$.

\subsection{Customer Satisfaction Index. (C.S.I)}

With VMI or with vendor managed forecasting we must check whether end customer is benefited or not. Other performance measures such as fill rates or cycle service levels, measure the proportion of customer demand that is satisfied from available inventory. It does not measure the satisfaction index of customers. Therefore, to measure satisfaction index we used the Customer Satisfaction Index as suggested by Sinha and Basu (1998). Manufacturer provided forecasted quantity to retailer. If the demand quantity is equal to the forecasted quantity then the event was HIT, otherwise MISS.

$$
\text { C.S.I. }=\text { Number of HIT/ Total number of orders }
$$

$D_{t}$ was the actual demand of customers and $F_{t}$ was the forecasted quantity for retailer.

CSI for the specific order was found as follows.

$$
\text { If } \quad F_{t} \geq D_{t}, C I_{t}=1 \text { otherwise zero }
$$


Hence, overall CSI $=\Sigma \mathrm{CSI}_{\mathrm{t}} /$ Total number of orders

\section{Results and Discussion}

It was observed that the change of policy suggested for year 2005-06, resulted in lower customer satisfaction index. As already discussed, we analyzed previous year's sales data and forecasted the demand of retailer for Year 2006-07. The objective of adopting vendor managed forecasting was to improve the customer satisfaction index and to reduce demand variation. We adopted various statistical forecasting techniques for predicting the demand. Expected demand from Winter's, Holt's, Fourier and Simple exponential model were forecasted using Excel 2008 software. Results are depicted in Table 2. It can be seen that, the forecast obtained from Winter's model would result in highest excess inventory proportions. Stocks out situations would be almost equal to those of Cyclical model. However, this model would provide highest customer satisfaction index and demand variance. In other words, if results of Winter's model were followed then there would have been serious wastage of inventory. As a result of over forecasting company could improve the customer satisfaction index but it would increase total supply chain cost. Results obtained from Simple Exponential model were far better than results of Winter's model. This model yielded minimum stock outs. Excess inventory proportions were also less than that of Winter's model, but customer satisfaction index was very low. Holt's model yields highest excess inventory and reduces stock outs considerably. However, this model doesn't improved customer satisfaction index.

Cyclical model improved customer satisfaction index. Lowest excess inventory proportions were observed for this model. Stock outs or short inventory proportions were also in line of other models. As compared to Holt 's, Fourier's and Winter's model customer satisfaction index was also improved. This means that Cyclical model provides trade-off of E.I, S.I and C.S.I. Thus, the duel objective of improving customer satisfaction index and reducing demand variation could be achieved with Cyclical model.

In the specialized literature, many authors have used statistical methodology to perform comparisons. We adopted Nemenyi's procedure for multiple pair wise comparisons of forecasts. This test posses low power; therefore in most of the experimentations it did not find any differences (García \& Herrera, 2008). 
However, in our study significant differences were found. Furthermore, in our research we found critical and significant differences for the results obtained from various forecasts. Bonferroni correction level was checked for stastistical significance. The Bonferroni correction is a safeguard against multiple tests of statistical significance on the same data falsely giving the appearance of significance. The probability of getting a significant result with $\mathrm{n}$ tests at this level of significance is $1-0.95^{n}$. Thus it indicates that results are not by chance. Using post hoc techniques, one cannot make specific comparisons, so we make all comparisons. The net effect is that we require a LARGER difference between means to call them significant (see, http://www.uwsp.edu/psych/cw/statmanual/posthocs.html). The results of comparisons for inventory levels are depicted in Table 3, Table 4, and Table 5. From Table 3 it can be seen that according to Nemenyi's procedure, Cyclical-forecasting Model outperforms the other models. Table 4 shows larger value of critical differences for Cyclical forecasting Model. Thus it is once again proved that the basic hypothesis that Cyclical Model would yield best results for our study is validated. However, Table 5 shows no significance difference for Bonferroni corrected significance level.

The most important thing in our study was measurement of forecasting accuracy. Lesser the value of absolute error, percentage error, mean absolute deviation better the forecast. Alon et al. (2001), Hsu et al. (2002), Chang et al. (2005), and Chang and Wang (2006) performed the similar study for monthly sales forecasting, with the purpose of improving the forecasting accuracy. Authors compared MAD, MAPE, and TS for various forecasting methods. In most of the studies, Winter's models and Exponential model exhibited a superior performance.

In our study, we have observed that past sales data had a seasonal component. Therefore, we believed on Cyclical forecaster for better results. It was felt that these vendor-managed forecasts would assist better coordination between supply chain members for inventory management decisions. Performance of each forecaster is presented in Table 6 and Table 10. Minimum Mean Absolute deviation and Mean Absolute Percent Error was for Cyclical forecaster while minimum Tracking signal range was for Holt model. It indicates that, if the supply chain decisions were taken on the basis of Cyclical forecaster there would be fewer sale failures and lesser inventory cost. An improvement in these performance indicators would also help company in improving customer base. Similar to previous studies, our study also found good results with exponential forecaster. 
Further, comparative analysis of forecasting accuracy was done to corroborate the choice of Cyclical forecaster. Multiple pair wise comparisons for forecasting accuracy using the Nemenyi's procedure is depicted in table 7.It can be seen that exponential forecaster is ranked first closely followed by Cyclical forecaster. Table 8 shows pair wise differences for forecasting accuracy. Once again larger differences are found for Cyclical forecaster. Table 9 revels significance difference for Bonferroni corrected significance level. It is found that only exponential forecaster and cyclical forecaster have significant differences. It indicates that results obtained are not by chance and we can have full confidence on results. Table 10 shows tracking signal range for various forecasts. For tracking signal the results of Winter and Holt model proved to be best as shown in table 11, 12. Thus, Cyclical Forecasting model failed on the criteria of tracking signal range.

The net effect of adoption of vendor-managed forecasts is reveled in Table 13. The improvement can be clearly seen. The demand variance is reduced to $24 \%$ only. It means the ratio of over and under forecasting has been reduced. Thus, there would be lesser stock outs as well as excess inventory situations. Additionally, direct effect of total supply chain cost can also be seen. Compared to previous year, total supply chain cost has been reduced. As a result, profit margins would be improved.

In 2005-06, to reduce the percentage of excess inventory company had adopted the policy of supplying less than what is demanded. But it resulted in very low customer satisfaction index. If ultimate customer is not satisfied then the company may lose the retailer. Adoption of vendor-managed forecasts in 2006-07,improved the customer satisfaction index to an acceptable level. As the retailer's customers were satisfied, retailer was also satisfied with the company. Thus adoption of vendor-managed forecasts was a win-win situation for retailer and company.

\section{Conclusions}

In this paper, we have presented a case study of a small scale Indian enterprise. It shows that even partial adoption of vendor-managed inventory helps to improve performance of a small enterprise. We have developed a simple information sharing mechanism between retailer and case industry. Using demand related information, vendor managed forecasts were developed. Case company followed this forecasts for production planning and inventory management decisions. Five statistical models were applied to forecast the demand. It was assumed that 
product being seasonal, Cyclical model would be best fitted for this study. To corroborate the proposition comparison with other models was evaluated. Results show that performance of Cyclical model is superior to other statistical models. Forecasts developed from this model allowed company to decrease inventory levels, reduce total supply chain cost and improve customer satisfaction simultaneously.

The findings of this study have important managerial implications. It is known that precise forecasting depends on forecasting horizons, information technology requirements and in-house expertise. Large company can easily take care of all this factors. However, for small enterprise it is difficult to invest in special software and support staff. Statistical forecasting models may not yield the forecast to the extent of special software. But the computation time, ease and associated cost is very less. Small and Medium enterprise needs to evaluate the trade off between cost and accuracy. Although this study showed that Cyclical model provided more accurate forecasts, company should evaluate the opportunity cost with other models.

We have considered a single product and a single tier supply chain. The results of this study can easily replicated with other small industries. However when the number of players and number of products increases it would be difficult to adopt the traditional models. The major limitation of this study is that we did not take into account other functions of VMI. Moreover traditional forecasting models does not take in to account the special case like, sales promotion, holidays, price variables etc. We intend to do future work in two directions. First, we would like to develop a hybrid model of Cyclical and Artificial neural network model that will take care of special cases and which may yield more precise results. Second, we want to adopt all features of vendor-managed inventory for improving the overall performance of a small-scale industry. 


\section{Appendices}

\begin{tabular}{|c|c|c|c|}
\hline Year & Excess I nventory & Short I nventory & Customer Satisfaction I ndex \\
\hline $\mathbf{2 0 0 4 - 0 5}$ & $22.27 \%$ & $2.59 \%$ & 0.771 \\
\hline $\mathbf{2 0 0 5 - 0 6}$ & $13.68 \%$ & $18.94 \%$ & 0.283 \\
\hline
\end{tabular}

Table 1. "Behavior of inventory and CSI for year 2004-05 and 2005-06"

\begin{tabular}{|c|c|c|c|c|c|}
\hline Measurel Model & Winter & Holt & Fourier & Exponential & Cyclical \\
\hline E.I . & 17.9 & 7.07 & 9.33 & 7.48 & 7 \\
\hline S.I . & 17.32 & 20.22 & 19.79 & 16.28 & 17.08 \\
\hline D.V & 34.41 & 27.29 & 92.12 & 23.76 & 24.08 \\
\hline C.S.I . & 0.4315 & 0.3406 & 0.3406 & 0.3076 & 0.4021 \\
\hline
\end{tabular}

Table 2. "Comparative statistics of inventory and CSI for year 2006-07"

\begin{tabular}{|l|c|c|c|c|}
\hline \multicolumn{1}{|c|}{ Sample } & Frequency & Sum of ranks & Mean of ranks & Groups \\
\hline Cyclical & 3 & 4.000 & 1.333 & $\mathrm{~A}$ \\
\hline Exponential & 3 & 6.000 & 2.000 & $\mathrm{~A}$ \\
\hline Holt & 3 & 10.500 & 3.500 & $\mathrm{~A}$ \\
\hline Fourier & 3 & 11.500 & 3.833 & $\mathrm{~A}$ \\
\hline Winter & 3 & 13.000 & 4.333 & $\mathrm{~A}$ \\
\hline
\end{tabular}

Table 3."Multiple pair wise for inventory comparisons using the Nemenyi's procedure"

\begin{tabular}{|l|c|c|c|c|c|}
\hline & Winter & Holt & Fourier & Exponential & Cyclical \\
\hline \multicolumn{1}{|c|}{ Winter } & 0 & 0.833 & 0.500 & 2.333 & 3.000 \\
\hline Holt & -0.833 & 0 & -0.333 & 1.500 & 2.167 \\
\hline Fourier & -0.500 & 0.333 & 0 & 1.833 & 2.500 \\
\hline Exponential & -2.333 & -1.500 & -1.833 & 0 & 0.667 \\
\hline Cyclical & -3.000 & -2.167 & -2.500 & -0.667 & 0 \\
\hline \multicolumn{6}{|r|}{ Critical difference: 3.6239 } \\
\hline
\end{tabular}

Table 4. "Pair wise differences for inventory results"

\begin{tabular}{|l|c|c|c|c|c|}
\hline & Winter & Holt & Fourier & Exponential & Cyclical \\
\hline Winter & No & No & No & No & No \\
\hline Holt & No & No & No & No & No \\
\hline Fourier & No & No & No & No & No \\
\hline Exponential & No & No & No & No & No \\
\hline Cyclical & No & No & No & No & No \\
\hline Bonferroni corrected significance level: 0.005 & \\
\hline
\end{tabular}

Table 5. "Significant differences for inventory results" 


\begin{tabular}{|l|c|c|c|c|c|}
\hline Measurel Model & Winter & Holt & Fourier & Exponential & Cyclical \\
\hline AE & 180 & 139 & 140 & 115 & 116 \\
\hline MAD & 212 & 160 & 161 & 131 & 116 \\
\hline PE & 48 & 34 & 36 & 31 & 31 \\
\hline MAPE & 56 & 36 & 39 & 33 & 34 \\
\hline
\end{tabular}

Table 6. "Comparative statistics of forecasting accuracy"

\begin{tabular}{|l|c|c|c|c|c|}
\hline \multicolumn{1}{|c|}{ Sample } & Frequency & Sum of ranks & Mean of ranks & \multicolumn{2}{c|}{ Groups } \\
\hline Exponential & 4 & 5.500 & 1.375 & $\mathrm{~A}$ & \\
\hline Cyclical & 4 & 6.500 & 1.625 & $\mathrm{~A}$ & \\
\hline Holt & 4 & 12.000 & 3.000 & $\mathrm{~A}$ & $\mathrm{~B}$ \\
\hline Fourier & 4 & 16.000 & 4.000 & $\mathrm{~A}$ & $\mathrm{~B}$ \\
\hline Winter & 4 & 20.000 & 5.000 & & $\mathrm{~B}$ \\
\hline
\end{tabular}

Table 7. “Multiple pair wise comparisons for forecasting accuracy using the Nemenyi's procedure"

\begin{tabular}{|c|c|c|c|c|c|}
\hline & Winter & Holt & Fourier & Exponential & Cyclical \\
\hline Winter & 0 & 2.000 & 1.000 & 3.625 & 3.375 \\
\hline Holt & -2.000 & 0 & -1.000 & 1.625 & 1.375 \\
\hline Fourier & -1.000 & 1.000 & 0 & 2.625 & 2.375 \\
\hline Exponential & -3.625 & -1.625 & -2.625 & 0 & -0.250 \\
\hline Cyclical & -3.375 & -1.375 & -2.375 & 0.250 & 0 \\
\hline \multicolumn{2}{|c|}{ Critical difference: 3.1384 } & & & \\
\hline
\end{tabular}

Table 8. "Pair wise differences forecasting accuracy"

\begin{tabular}{|l|c|c|c|c|c|}
\hline & Winter & Holt & Fourier & Exponential & Cyclical \\
\hline Winter & No & No & No & Yes & Yes \\
\hline Holt & No & No & No & No & No \\
\hline Fourier & No & No & No & No & No \\
\hline Exponential & Yes & No & No & No & No \\
\hline Cyclical & Yes & No & No & No & No \\
\hline Bonferroni corrected significance level: 0.005 & & \\
\hline
\end{tabular}

Table 9. "Significant differences for forecasting accuracy" 


\begin{tabular}{|l|c|c|c|c|c|}
\hline T.S Range\ Model & Winter & Holt & Fourier & Exponential & Cyclical \\
\hline From & -20 & -38 & -63 & -137 & -73 \\
\hline To & 65 & 15 & 260 & 23 & 74 \\
\hline
\end{tabular}

Table 10. "Tracking signal range"

\begin{tabular}{|l|c|c|c|c|}
\hline \multicolumn{1}{|c|}{ Sample } & Frequency & Sum of ranks & Mean of ranks & Groups \\
\hline Exponential & 2 & 3.000 & 1.500 & $\mathrm{~A}$ \\
\hline Holt & 2 & 5.000 & 2.500 & $\mathrm{~A}$ \\
\hline Cyclical & 2 & 6.000 & 3.000 & $\mathrm{~A}$ \\
\hline Winter & 2 & 8.000 & 4.000 & $\mathrm{~A}$ \\
\hline Fourier & 2 & 8.000 & 4.000 & $\mathrm{~A}$ \\
\hline
\end{tabular}

Table 11. “Multiple pair wise comparisons for tracking signal range using the Nemenyi's procedure"

\begin{tabular}{|c|c|c|c|c|c|}
\hline & Winter & Holt & Fourier & Exponential & Cyclical \\
\hline Winter & 0 & 1.500 & 0.000 & 2.500 & 1.000 \\
\hline Holt & -1.500 & 0 & -1.500 & 1.000 & -0.500 \\
\hline Fourier & 0.000 & 1.500 & 0 & 2.500 & 1.000 \\
\hline Exponential & -2.500 & 1.000 & -2.500 & 0 & -1.500 \\
\hline Cyclical & -1.000 & 0.500 & -1.000 & 1.500 & 0 \\
\hline \multicolumn{3}{|c|}{ Critical difference: 4.4383} & & & \\
\hline
\end{tabular}

Table 12. "Pair wise differences for tacking signal"

\begin{tabular}{|c|c|c|c|}
\hline Measure & $\mathbf{2 0 0 4 - 0 5}$ & $\mathbf{2 0 0 5 - 0 6}$ & $\mathbf{2 0 0 6 - 0 7}$ \\
\hline Demand Variance & $25 \%$ & $38 \%$ & $24 \%$ \\
\hline Inventory Cost & 813051 & 708645 & 697749 \\
\hline CSI & 0.771 & 0.283 & 0.4021 \\
\hline
\end{tabular}

Table 13. "Effects of VMI adoption" 


\section{References}

Alon, I., Qi, M., \& Sadowski, R.J. (2001). Forecasting aggregate retail sales: a comparison of artificial neural networks and traditional methods. Journal of Retailing and Consumer Services, 8, 147-156.

Biegel, J.E. (1987). Production Control - A Quantitative Approach, Prentice Hall India Ltd., Delhi.

Box, G.E.P., Jenkins, G.M., \& Reinsel, G.C. (2004). Time Series Analysis Forecasting and Control. Pearson Education Asia: Delhi.

Chang, P.C., Wang, Y.W., \& Tsai, C.Y. (2005). Evolving neural network for printed circuit board sales forecasting. Expert System with Applications, 29, 83-92.

Chang, P.C., Lai, C.Y., \& Lai K.R. (2006). A hybrid system by evolving case-based reasoning with genetic algorithm in wholesaler's returning book. Decision Support Systems, 42, 1715-1729.

Chang, P.C., \& Wang, Y.W. (2006). Fuzzy Delphi and back- propagation model for sales forecasting in PCB industry. Expert Systems with Applications, 30, 715-726.

Chang, K.K. (2005). Applying Fourier Transform and Linear Time Invariant Analysis to Brain Imaging Data, Project for Machine Learning. Retrieved September $10^{\text {th }}$ from www.cs.cmu.edu/ kkchang/paper/Chang.2005.10-701.brain/maging.rtf

Chopra, S., \& Meindl, P. (2001). Supply Chain management: Strategy, Planning and Operation. Pearson Education: Asia, Delhi.

Chu, C.W., \& Zhang, G.P. (2003). A comparative study of linear and nonlinear models for aggregate retail sales forecasting. International Journal of Production Economics, 86, 217-231.

Dekker, M., Donselaar, K., \& Ouwehand, P. (2004). How to use aggregation and combined forecasting to improve seasonal demand forecasts. International Journal Production Economics, 90, 151-167.

Disney, S.M., \& Towill, D.R. (2003). Vendor-managed inventory and bullwhip reduction in a two-level supply chain. International Journal of Operations \& Production Management, 23 (6), 625-651 
Frees, E.W., \& Miller, T. W. (2004). Sales forecasting using longitudinal data models. International J ournal of Forecasting, 20, 99-114.

Garcia, S., \& Herrera, F. (2008). An Extension on "Statistical Comparisons of Classifiers over Multiple Data Sets" for all Pairwise Comparisons. Journal of Machine Learning Research, 9, 2677-2694.

Harrison, P.J. (1965). Short term demand forecasting. Applied Statistics, 14, 102139.

Haq, A., \& Kannan, G. (2006). Effect of forecasting on the multi echelon distribution inventory supply chain cost using neural network, genetic algorithm and particle swarm optimization. International J ournal of Services Operations and Informatics, $1(1 / 2), 1-22$.

Holt, C.C., Modigliani, F.J., Muth, F., \& Simon, H.A. (1963). Planning Production, Inventories and Work Force, Prentice Hall, Englewood Cliffs, N.J .

Hsu, P.H., Wang, C.H., Shyu, J.Z., \& Yu, H.C.(2002). A litterman BVAR approach for production forecasting of technology industries. Technological Foresating and Social Change, 70, 67-82.

Jung, S., Chang, T.W, Sim, E., \& Parl, J. (2004). Vendor Managed Inventory and Its Effect in the Supply Chain , Asia Sim LNAI 3398, 545-552

Krajewski, L.J., \& Ritzman,R.P. (2000). Operations Management Strategy and Analysis. Pearson Education Asia: Delhi.

Kurawarwala, A.A., \& Matsuo, H. (1998). Product growth models for medium-term forecasting of short life cycle products. Technological Forecasting and Social Change, 57, 169-196.

Lee, H.L., \& Whang, S. (2000). Information sharing in a supply chain. International Journal of Technology Management, 20, 373-387.

Raveh, A., \& Tapiero, C.S. (1980). Finding common seasonal patterns among time series. Journal of Econometrics, 12, 353-363

Rudberg, M., Klingenberg, M., \& Kronhamn, K. (2002). Collaborative supply chain planning. Integrated Manufacturing System, 13 (8), 596-610. 
Sharda, R., \& Patil, R. (1992). Connectionist approach to time series prediction; an empirical test. Journal of Intelligent Manufacturing, 3, 317-323.

Sharma, M.K., \& Bhagwat, R. (2005). Practice of information systems Evidence from select Indian SMEs. Journal of Manufacturing Technology Management, $17(2), 199-223$.

Sinha, R.K., \& Basu, S. (1998). Quality of customer service in supply chain system: a diagnostic study. International Journal of Quality and Reliability Management, 15 (9/9), 844-859.

Snyder, R.D, Koehler, A.B., Hyndman, R.J., \& Or, J.K. (2004). Exponential smoothing models: Means and variances for lead-time demand .European Journal of Operational Research, 158, 444-455.

Thomassey, S.T., \& Fiordaliso, A. (2006). A hybrid sales forecasting system based on clustering and decision trees. Decision Support Systems, 42, 408- 421.

Tseng , F.M., Yu, H.C., \& Tzeng, G.H. (2001). Applied hybrid grey model to forecast seasonal time series. Technological Forecasting and Social Change, 67, 291-302.

Tyan J., \& Wee, H. (2003). Vendor managed inventory: a survey of the Taiwanese grocery industry. Journal of Purchasing \& Supply Management, 9(1), 11-18

Wang, T.Y., \& Huang, C.Y. (2007). Improving forecasting performance by employing the Taguchi method. European Journal of Operational Research, 176, 1052- 1065.

Winklhofera, H., \& Diamantopoulos, A. (2003). A model of export sales forecasting behaviour and performance: development and testing. International Journal of Forecasting, 19, 271-285.

Yelland, P.M. (2006). Stable seasonal pattern models for forecast revision: A comparative study. International J ournal of Forecasting, 22, 799-818.

Zhao, X., Xie, T., \& Zhang, W.J. (2003). The impact of information sharing and ordering co-ordination on supply chain performance. Supply Chain Management An International J ournal, 7(1), 24-40. 
Zhifang L., Guoqiao R., \& Xiaoting S. (2006). Research of chaotic SVM with incorporated intelligence algorithm forecasting model in SCM. International Journal of Computer Science and Network Security, 6(10), 136 -141

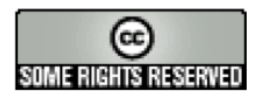

Article's contents are provided on a Attribution-Non Commercial 3.0 Creative commons license. Readers are allowed to copy, distribute and communicate article's contents, provided the author's and Journal of Industrial Engineering and Management's names are included. It must not be used for commercial purposes. To see the complete license contents, please visit http://creativecommons.org/licenses/by-nc/3.0/. 